\title{
II. CARBON AND CARbONATE ANALYSES, DEEP SEA DRILLING PROJECT LEG 50
}

\author{
Gerald W. Bode, Scripps Institution of Oceanography, La Jolla, California
}

Leg 50 sediments were analyzed for total carbon and acid-insoluble (organic) carbon, using the new Leco WR-12 Analyzer according to the standard technique outlined below. The reproducibility of the Leco was verified in extensive tests prior to Leg 50, and also in standards run for subsequent legs.

The $3-\mathrm{cm}^{3}$ sediment samples were first dried and ground into a homogeneous powder. The ground sediment was redried at 105 to $110^{\circ} \mathrm{C}$, and two samples, a 0.1 -gram and a 0.5 -gram sample, were weighed into Leco clay crucibles. The 0.5 -gram sample was acidified with 10 per cent hydrochloric acid and washed with distilled water. The sample was then dried and analyzed for acid-insoluble carbon. The 0.1-gram sample was analyzed for total carbon, without further treatment. If the sample contained less than 10 per cent $\mathrm{CaCO}_{3}$, an additional 0.5 -gram sample was analyzed for greater accuracy. The calcium-carbonate percentages were calculated as follows: ( $\%$ total $\mathrm{C}-\%$ organic $\mathrm{C}) \times$ $8.33=\% \mathrm{CaCO}_{3}$. Although other carbonates may be present, all acid-soluble carbon was calculated as calcium carbonate. All results are given in weight percent (Table 1).

Detailed descriptions of the technique and theory may be found in Bader, Gerard, et al. (1970) and Boyce and Bode (1972).

TABLE 1

Leg 50 Carbon and Carbonate Analyses

\begin{tabular}{ccccc}
\hline & $\begin{array}{c}\text { Sub- } \\
\text { bottom } \\
\text { Depth } \\
\text { Core } \\
\text { of Sample } \\
(\mathrm{m})\end{array}$ & $\begin{array}{c}\text { Total } \\
\text { Carbon } \\
(\%)\end{array}$ & $\begin{array}{c}\text { Organic } \\
\text { Carbon } \\
(\%)\end{array}$ & $\begin{array}{c}\mathrm{CaCO}_{3} \\
(\%)\end{array}$ \\
\hline 415-1-1 & 0.17 & 7.1 & 0.2 & 58 \\
$2-1$ & 75.01 & 8.2 & 0.1 & 68 \\
$3-1$ & 138.38 & 8.1 & 0.2 & 66 \\
$4-1$ & 207.41 & 9.0 & 0.1 & 74 \\
$415 \mathrm{~A}-5-1$ & 443.34 & 0.4 & 0.3 & 0 \\
$9-4$ & 647.54 & 5.5 & 0.9 & 38 \\
$415 \mathrm{~B}-2-1$ & 57.40 & 8.8 & 0.1 & 73 \\
$416 \mathrm{~A}-10-2$ & 1187.76 & 1.4 & 0.4 & 8 \\
$10-2$ & 1187.77 & 1.5 & 0.1 & 12 \\
$10-2$ & 1187.79 & 4.6 & 0.9 & 31 \\
$10-2$ & 1187.82 & 4.6 & 0.9 & 31 \\
$10-2$ & 1187.84 & 3.0 & 0.3 & 22 \\
$10-2$ & 1187.85 & 4.2 & 0.1 & 35 \\
$11-1$ & 1194.92 & 4.1 & 0.5 & 30 \\
$11-1$ & 1195.03 & 0.9 & 0.5 & 3 \\
$11-1$ & 1195.40 & 2.5 & 0.1 & 20 \\
$13-1$ & 1214.27 & 3.0 & 0.6 & 20 \\
\hline
\end{tabular}

TABLE 1 - Continued

\begin{tabular}{|c|c|c|c|c|}
\hline $\begin{array}{l}\text { Core } \\
\text { Section }\end{array}$ & $\begin{array}{c}\text { Sub- } \\
\text { bottom } \\
\text { Depth } \\
\text { of Sample } \\
\text { (m) }\end{array}$ & $\begin{array}{c}\text { Total } \\
\text { Carbon } \\
(\%)\end{array}$ & $\begin{array}{c}\text { Organic } \\
\text { Carbon } \\
(\%)\end{array}$ & $\begin{array}{c}\mathrm{CaCO}_{3} \\
(\%)\end{array}$ \\
\hline $13-1$ & 1214.36 & 1.4 & 0.1 & 11 \\
\hline $13-1$ & 1214.41 & 4.1 & 0.7 & 29 \\
\hline $16-1$ & 1243.75 & 7.6 & 0.5 & 59 \\
\hline $16-3$ & 1245.78 & 0.4 & 0.4 & 0 \\
\hline $17-1$ & 1252.45 & 3.8 & 0.6 & 27 \\
\hline $19-5$ & 1277.63 & 2.6 & 0.5 & 18 \\
\hline $19-5$ & 1277.77 & 6.4 & 0.7 & 47 \\
\hline $19-5$ & 1277.93 & 1.0 & 0.4 & 5 \\
\hline $21-1$ & 1290.80 & 1.1 & 0.4 & 6 \\
\hline $21-1$ & 1291.10 & 2.2 & 0.4 & 15 \\
\hline $21-3$ & 1294.26 & 0.8 & 0.2 & 5 \\
\hline $21-5$ & 1297.11 & 5.1 & 0.4 & 39 \\
\hline $23-4$ & 1313.90 & 6.6 & 1.0 & 47 \\
\hline $23-4$ & 1314.21 & 1.7 & 0.5 & 10 \\
\hline $23-4$ & 1314.37 & 3.4 & 0.6 & 23 \\
\hline $25-2$ & 1329.40 & 1.4 & 0.4 & 9 \\
\hline $25-2$ & 1329.60 & 2.4 & 0.4 & 17 \\
\hline $25-3$ & 1331.06 & 6.8 & 0.4 & 54 \\
\hline $27-1$ & 1347.91 & 0.7 & 0.6 & 2 \\
\hline $27-2$ & 1348.89 & 3.5 & 0.3 & 27 \\
\hline $27-3$ & 1350.76 & 4.5 & 0.3 & 35 \\
\hline $28-2$ & 1358.46 & 6.8 & 0.8 & 50 \\
\hline $28-4$ & 1360.97 & 0.7 & 0.1 & 5 \\
\hline $29-3$ & 1369.19 & 8.0 & 0.3 & 64 \\
\hline $29-4$ & 1371.40 & 1.8 & 0.3 & 13 \\
\hline $35-1$ & 1416.76 & 7.9 & 0.2 & 64 \\
\hline $35-1$ & 1417.14 & 1.2 & 0.6 & 5 \\
\hline $36-3$ & 1428.73 & 4.6 & 0.1 & 37 \\
\hline $38-1$ & 1445.34 & 7.7 & 0.1 & 63 \\
\hline $38-1$ & 1445.56 & 2.4 & 0.1 & 20 \\
\hline $38, \mathrm{CC}$ & 1447.06 & 6.7 & 0.1 & 55 \\
\hline $42-3$ & 1476.43 & 1.2 & 0.1 & 9 \\
\hline $44-1$ & 1492.52 & 1.1 & 0.2 & 7 \\
\hline $45-1$ & 1502.20 & 1.6 & 0.1 & 12 \\
\hline $45-2$ & 1503.82 & 7.9 & 0.1 & 65 \\
\hline $47-2$ & 1522.49 & 10.5 & 0.1 & 87 \\
\hline $48-2$ & 1531.07 & 1.5 & 0.1 & 12 \\
\hline $50-2$ & 1550.24 & 10.9 & 0.0 & 9 \\
\hline $52-1$ & 1567.96 & 0.9 & 0.1 & 7 \\
\hline
\end{tabular}

\section{REFERENCES}

Bader, R. G., Gerard, R. D., et al., 1970. Initial Reports of the Deep Sea Drilling Project, v. 4: Washington (U. S. Government Printing Office).

Boyce, R. E., and Bode, G. W., 1972. Carbon and carbonate analyses, Leg 9, Deep Sea Drilling Project. In Hayes, J. D., et al., Initial Reports of the Deep Sea Drilling Project, v. 9: Washington (U. S. Government Printing Office), p. 747. 